Journal of Business and Management Studies (JBMS)

ISSN: 2709-0876

DOI: $10.32996 / j b m s$

Journal Homepage: www.al-kindipublisher.com/index.php/jbms

\title{
Insertion des femmes diplômées d'universités dans milieu professionnel à Lubumbashi en République démocratique du Congo
}

\author{
Ilunga Kalombo Fifi ${ }^{1 *}$ and Sem Mbimbi Pascal ${ }^{2}$ \\ ${ }^{12}$ Université de Lubumbashi, Faculté des Sciences Economiques et de Gestion \\ Corresponding Author: Ilunga Kalombo Fifi, E-mail: Ilungakalombofifi@gmail.com
}

ARTICLE INFORMATION

Received: November 02, 2020

Accepted: January 01, 2021

Volume: 3

Issue: 1

DOI: 10.32996/jbms.2021.3.1.1

\section{KEYWORDS}

Insertion professionnelle, Travail, Femmes diplômées, Chômage, Lubumbashi

\section{ABSTRACT}

La République démocratique du Congo en général et la ville de Lubumbashi en particulier enregistrent une croissance démographique rapide accompagnée d'énormes défis socio-économiques. Alors que le chômage ainsi que les inégalités sociales sont largement documentés, cette situation touche malheureusement plus des femmes malgré leurs niveaux d'étude universitaire. À Lubumbashi, l'enquête conduite dans les entreprises publiques et privées renseigne que seulement $9 \%$ des femmes universitaires y travaillent dans l'administration malgré le nombre important de femmes diplômées ces dernières années. L'objectif de cette étude était d'identifier les facteurs d'insertion professionnelle ainsi que des défis à relever pour capitaliser, au profit du pays, les compétences des femmes universitaires pour une croissance plus inclusive. Les données utilisées dans le cadre de cette étude proviennent d'une enquête conduite à Lubumbashi auprès de 384 femmes diplômées d'universités choisies de manière aléatoire en 2019. Les résultats indiquent $76 \%$ de femmes interrogées ont obtenu leurs licences mais celles qui ont fait leurs études dans des filières spécialisées (par exemple : médecine, accouchement) et des filières techniques (par exemple : géologie, chimie) se sont vite insérées dans le monde professionnel. En outre, la matrice de corrélation montre que la position du mari dans une entreprise et sa rémunération, le statut des parents, la connaissance de la langue anglaise ainsi que de l'informatique sont des éléments supplémentaires positivement corrélées à l'insertion professionnelle des femmes diplômées à Lubumbashi. Ces résultats nous motivent d'inviter et d'encourager les filles à s'inscrire dans les filières techniques et apprendre l'outil informatique et l'anglais pour augmenter les chances de trouver de l'emploi. Cette étude constitue aussi une source d'informations fiables pour le pouvoir public qui cherche à améliorer les conditions d'accès des femmes au marché du travail.

\section{Introduction}

La République démocratique du Congo enregistre une croissance démographique rapide accompagnée d’énormes défis socio-économiques (Balasha et Nkulu, 2020).De 1960 à 2000, sa population est passée de 15.248.258 à 47.105 .826 d'habitants jusqu'à frôler les 87 millions d'habitants en 2019 (Figure 1). Cette population majoritairement jeune, composée d’hommes et des femmes (voir annexe, Figure 1) constitue une opportunité de développement pour le pays.

\section{K C AL-KINDI CENTER} $\mathbf{R}$ D FER RESEARCH Your gatewoy to world-class research
Published by Al-KindiCenter for Research and Development. Copyright (c) the author(s). This is an open access article under CC BY license (https://creativecommons.org/licenses/by/4.0/) 


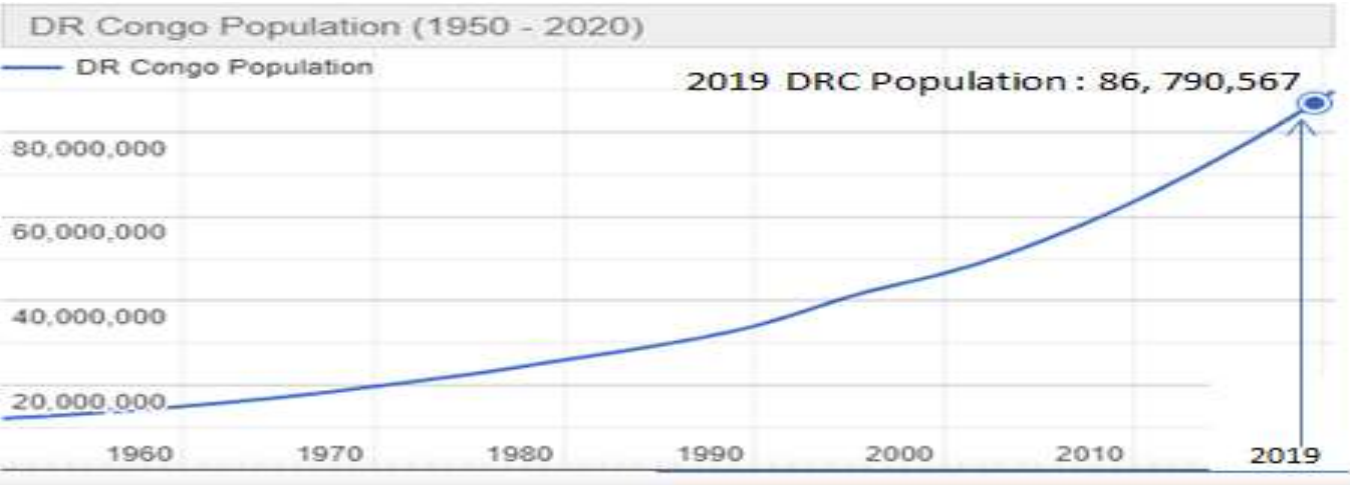

Figure 1. Evolution démographique en RD Congo entre1960-2019²

Selon Doumbia et Kuépié (2008), les ressources humaines abondantes dans nombreux pays africains peuvent constituer un facteur de croissance si des politiques économiques appropriées permettent de mobiliser dans des activités productives toute la main-d'œuvre potentielle. La RD Congo a besoin de faire pleinement usage des compétences et des talents de tous ses citoyens (hommes et femmes) afin d'assurer une croissance plus inclusive et plus équilibrée, pour consolider sa croissance et d'accéder à l'émergence (Mellor, 2014). Cependant, face à la conjoncture actuelle du marché du travail, l'insertion professionnelle et l'autonomisation des femmes est devenue une problématique réelle, tant sur le plan national que sur le plan local. Selon Betasemi (2018), le travail donne l'identité à l'homme, contribue à son statut social, et dans une autre mesure et à sa dignité car ce dernier apparaît comme le moyen privilégié d'avoir accès aux biens et services disponibles dans la vie. Le 8 mars 2018 à l'occasion de la journée internationale de la femme, le ministre congolais de l'Emploi-Genre et Famille, son excellence madame Lukiana Mufwankolo ${ }^{2}$ déclarait que seuls 2 à $4 \%$ des femmes actives peuvent trouver un emploi dans le secteur structuré, alors que le reste se débrouille dans les activités précaires ${ }^{3}$. Les inégalités ainsi que la sousreprésentation des femmes s'observent aussi dans le paysage politique en RD Congo même si la participation des femmes à la gestion de la chose publique est constitutionnelle. A titre illustratif, en 2010, la proportion des femmes à l'Assemblée nationale et au Sénat a été respectivement de $8.4 \%$ et $4.6 \%$ (JICA ,2017). Pourtant, JICA (2017) et Moummi(2010) insistent sur la nécessité de réduire les inégalités entre les hommes et les femmes quant à l'accès aux postes de responsabilité, aux ressources, à l'instruction et au travail pour permettre une croissance plus inclusive. On estime à plus de $80 \%$, la proportion de femmes qui travaille dans le secteur informel (Balasha et al., 2020 ; Murhula et al., 2020), dans l'agriculture familiale (Balasha et Nkulu, 2020). D’autres travaillent dans les structures informelles où elles sont mal payées et non protégées par la législation du travail ou par les normes sociales et n'ont pas d'avantages sociaux (assurance maladie par exemple ; congés) ni de salaire précis (lyenda, 2005 ; MONUSCO, 2019). Face à ces inégalités, l'accès à l'éducation universitaire par les femmes devrait, en principe être comme un facteur clé d'insertion professionnelle et de réussite (bon travail, bon salaire, stabilité sociale) et de positionnement (poste de responsabilité) dans la société. Alors qu'à Lubumbashi, les cinq dernières années les institutions supérieures et universitaires ont diplômé plus de 36,6\% des femmes (tableau 1), très peu d'elles (moins de 10\% de ces femmes) ont été insérées dans la vie professionnelle (tableau 2).

Tableau 1. Proportion en pourcentage des femmes et des hommes diplômés de 2014-2018

\begin{tabular}{lcc}
\hline Institutions & Femmes & Hommes \\
\hline Université de Lubumbashi & 26 & 74 \\
Institut Supérieur de Commerce de Lubumbashi & 41 & 59 \\
Institut Supérieur Pédagogique de Lubumbashi & 20 & 80 \\
Institut Supérieur d'Études Sociales & 38 & 62 \\
Institut Supérieur des Techniques Médicales & 54 & 46 \\
École Supérieure d'Informatique Salama & 43 & 57 \\
Université Protestante de Lubumbashi & 34 & 66 \\
\hline Proportion moyenne en pourcentage & $\mathbf{3 6 , 6}$ & $\mathbf{6 3 , 4}$ \\
\hline
\end{tabular}

Source : notre enquête sur terrain, 2019

\footnotetext{
${ }^{2}$ https://www.worldometers.info/world-population/democratic-republic-of-the-congo-population/

${ }^{3}$ ww.francetvinfo.fr/monde/afrique/politique-africaine/8mars2018 .
} 
Pourtant, avant les années 90 , le passage de l'université à la vie professionnelle s'effectuait pour la grande majorité des jeunes, sans problème particulier, de manière quasi instantanée. Ils rejoignaient la fonction publique ou les entreprises étatiques ou paraétatiques. Cette situation de "passage" préprogrammé de l'insertion professionnelle a presque disparu au cours des années 90 suite à l'avènement de pillage et de trouble politique qui s'en est suivi ainsi que la faillite de la Gécamines et de la SNCC (Nkuku, M. Rémon,2006;Tambwe,2010). Dans le même sens, l'étude de Nzuzi (2014) montre que I'instabilité politique qu'a connue la RDC, les pillages des années 1991 et 1993 ainsi que les conflits armés de 1998 à 2002 ont affecté négativement le marché de l'emploi. La crise financière de 2008 quant à elle, a aussi conduit à la fermeture des entreprises et à la compression des effectifs dans certaines entreprises en RDC en général et dans la province du Katanga en particulier. Cette situation a eu comme conséquences la perte d'emploi de plusieurs milliers des travailleurs katangais et par la suite la dégradation des conditions de vie de leurs ménages (Kyamakosa et Musewa, 2011). Nombreuses stràtegies ont été developpées pour survivre ${ }^{4}$. Lubumbashi est une ville minière potentiellement riche, siège de beaucoup d'institutions tant privées que publiques. Le taux d'insertion des femmes dans la vie professionnelle y est trop faible : seuls $9 \%$ des femmes diplômées universitaire travaillent dans l’administration et les entreprises publiques et privées (Tableau 2).

Tableau 2. Insertion professionnelle des femmes diplômées universitaires

\begin{tabular}{lll}
\hline Institutions & \% Femmes & \% Hommes \\
\hline Publiques & $13,8 \%$ & $86,2 \%$ \\
\hline Privées & $8,8 \%$ & $91,2 \%$ \\
\hline Taux d'insertion professionnelle & $9,1 \%$ & $90,9 \%$ \\
\hline
\end{tabular}

Source : notre enquête sur terrain,2019

C'est suite à cette disparité flagrante, illustrée par une infériorité numérique de femmes dans les entreprises que nous avons décidé d'amorcer cette étude avec une question principale de recherche. Quels sont les déterminants d'insertion des femmes diplômées d'universités dans la vie professionnelle à Lubumbashi ?

Pour répondre à cette question, une enquête a été menée auprès des femmes universitaires et nous avons couplé cette enquête à une analyse des théories existantes sur l'insertion professionnelles des femmes.

L'objectif de cette étude était d'identifier les facteurs d'insertion professionnelle ainsi que des défis à relever pour capitaliser les compétences des femmes universitaires pour un développement plus inclusif. Les objectifs spécifiques pours uivis sont : (1) décrire les caractéristiques sociodémographiques des femmes universitaires enquêtées (2) analyser les déterminants d'insertion des femmes diplômées d'universités dans la vie professionnelle à Lubumbashi .Cette étude peut être une source crédible d'information pour le pouvoir public et ses partenaires au développement qui cherchent à améliorer les conditions d'accès des femmes au marché du travail car celles-ci ont du potentiel, des compétences ainsi du savoir-faire à mettre au service de la nation.

\section{Théories de l'insertion professionnelle des femmes}

Les études sur le marché du travail ont montré qu'un peu partout dans le monde, à des degrés divers, les femmes occupent des positions désavantageuses. Plusieurs théories explicatives ont été développées pour cerner les différences entre hommes et femmes sur le marché du travail. Doumbia et Kuépié (2008) ont identifié trois théories dans le cadre de leur étude sur les déterminants de l'insertion des femmes sur le marché du travail (1) la théorie des stratégies de survie qui postule que la participation des femmes est fortement liée aux contraintes de survie des ménages; (2) la théorie du capital humain qui lie la participation et la position d'un individu sur le marché du travail au stock de capital humain accumulé et (3) les théories féministes qui montrent que la position défavorable des femmes sur le marché du travail n'est qu'un prolongement de leur situation dans la société. Nombreux chercheurs ont fondé leurs réflexions sur la théorie cognitive sociale. Cette théorie stipule que le sentiment de compétence influe sur les buts, à savoir que plus on se sent compétente, plus on peut se fixer des buts et des objectifs à atteindre. D'après Armstrong et Crombie (2000), les filles expriment un sentiment de compétence plus élevé pour les métiers "féminins" (soins infirmiers, social, éducation, etc.), alors que les garçons témoignent d'un sentiment de compétence plus élevé pour les métiers traditionnellement "masculins " (métier d'ingénieur par exemple). Pour l'évaluation des capacités individuelles dans le monde professionnel, la théorie d'autocensure a été également considérée. Celle-ci stipule que ce sont des sentiments internes soit de culpabilité et des croyances individuelles ou d'infériorité qui limitent nombreuses femmes à exprimer leur potentiel. Au regard de ces théories exploitées et l'observation rapprochée de notre société, cette étude sera axée sur l'hypothèse selon laquelle l'insertion des femmes diplômées d'universités dans le

${ }^{3}$ Pour améliorer les conditions de vie précaires et ainsi épauler leurs maris sans emploi, certaines femmes au Katanga ont développé des stratégies de survie dans le seul but de venir au secours à leur famille en apportant une contribution financièrement substantielle. 
milieu professionnel à Lubumbashi est expliquée par le capital humain et familiales ainsi que par la spécialité de formation de la femme. Des facteurs socio-culturels, économique et des perceptions individuelles peuvent constituer des barrières pour la vie professionnelle de la femme.

\section{Cadre d'Étude et Approches Méthodologiques}

\subsection{Cadre d'Étude}

Cette recherche sur l'Insertion professionnelle des femmes diplômées universitaires s'est réalisée au Sud -Est de la RDC, dans la province du Haut-Katanga, ville de Lubumbashi (Figure 2).

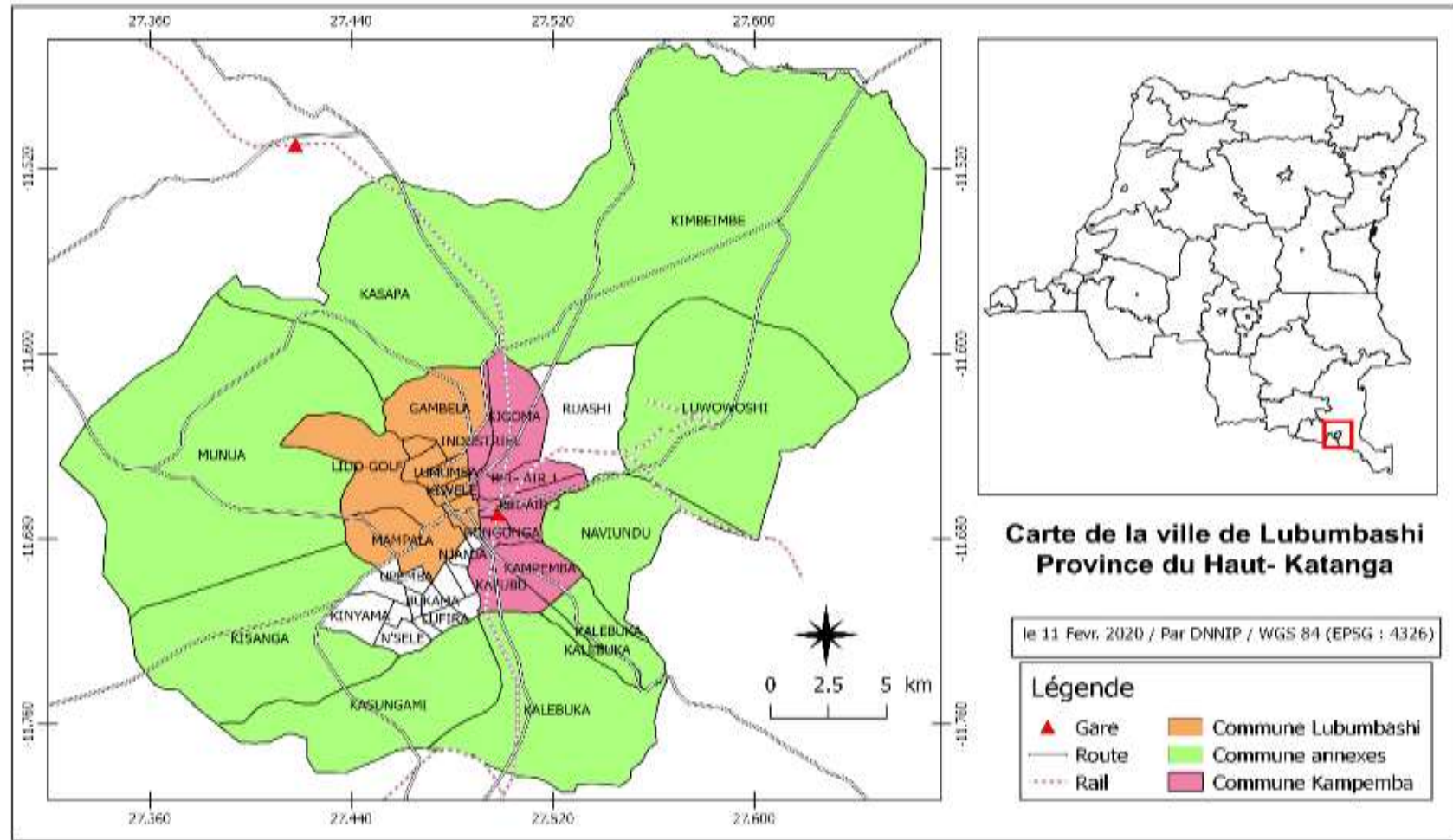

Figure 2. Ville de Lubumbashi et ses sept communes, RDC, carte tirée de Tshomba et al., 2020

La RDC est parmi les pays les plus pauvres du monde avec un indice du développement humain faible(PNUD ,2014). Sa population est très jeune (l'âge médian est de 17 ans) et le taux de chômage est autour de $90 \%$. La ville de Lubumbashi s'étend sur 7 communes: Lubumbashi, Kenya, Kampemba, Katuba, Kamalondo, Ruashi et Annexe (Nkuku, M. Rémon 2006). En 2016, Lubumbashi comptait déjà 2097000 habitants (UN, 2016) avec un taux annuel de croissance démographique d'environ 5\% (Useni et al., 2018). La demande en services dans plusieurs secteurs n'a cessé d'augmenter. Par exemple, dans le domaine d'éducation, Lubumbashi compte une université publique (Université de Lubumbashi) et plusieurs instituts supérieurs publics et privés (voir tableau 1).

\subsection{Approches Méthodologiques}

Les données utilisées dans le cadre de cette étude proviennent d'une enquête conduite à Lubumbashi auprès de 384 femmes universitaires choisies d'une manière aléatoire. Les femmes enquêtées sont issues de tous les niveaux de formation universitaire et font partie de nombreux jeunes qui ont quitté l'université il y a plus de 5 ans. A l'instar de l'étude de Calmand et Epiphane(2012), cette étude a privilégié une approche longitudinale de l'insertion. Elle permet à chaque femme enquêtée de retracer son parcours professionnel, depuis sa sortie du système éducatif, de 2013 jusqu'en 2019 soit environ 7ans. Le questionnaire administré comprenait les rubriques suivantes: caractéristiques sociodémographiques, emploi, chômage, études faites, situation des parents et du conjoint. Les femmes enquêtées ont été rencontrées dans leurs ménages entre 14heures et 18heures. Outre l'enquête, les théories sur l'insertion professionnelles ont été exploitées pour comprendre les enjeux, les défis et les aspirations des femmes dans le monde professionnel. 
Pour analyser les données, les statistiques descriptives effectuées des fréquences et des pourcentages résument les données relatives aux caractéristiques sociodémographiques. Une analyse par correspondance multiple $(\mathrm{acm})$ a été utilisée pour mettre en évidence des variables résumant le maximum d'information, en regroupant tous les éléments dans des classes homogènes c'est-à-dire les éléments qui s'associent. Le choix de l'acm est qu'elle permet de combiner les variables quantitatives et les variables qualitatives. Une matrice de corrélation a été élaborée pour déterminer les variables associées positivement à l'insertion professionnelle des femmes enquêtées. Une régression multiple a été utilisée pour identifier les facteurs plus déterminants de l'insertion professionnelle des femmes à Lubumbashi.

\section{Résultats et Discussion}

\subsection{Caractéristiques sociodémographiques des enquêtées}

Le tableau 3 porte sur les caractéristiques sociodémographiques des enquêtées. Une large partie (49\%) des répondantes a un âge qui varie entre 36 et 45 ans ; 24\% sont âgés entre 26 et 35 ans et seuls $3 \%$ ont plus de 56 ans. Ces jeunes femmes sont majoritairement célibataires (62\%) même si la proportion de mariées est aussi importante, s'élevant ainsi à $33 \%$. La majorité de ces enquêtées ont obtenu leurs licences $(76 \%)$ ou un graduat $(22 \%)$ dans une institution supérieure ou universitaire (tableau 1). Cependant, on note une très faible proportion de femmes ayant obtenu un master ou un doctorat. Cette faible proportion est également observée pour les filières techniques appliquées (ex: polytechnique, géologie, agronomie) ou spécialisées (médecine, pédiatrie). A peu près la moitié soit $49 \%$ des femmes enquêtées ont obtenu leurs diplômes universitaires il y a 5 à 10 ans, pourtant, jusqu'à la période de cette enquête, nombreuses de répondantes ont déclaré n'avoir pas encore trouvé de l'emploi. Plus de $60 \%$ de femmes qui étaient déjà insérées dans la vie professionnelle ont suivi à l'université les filières techniques ou spécialisées.

Tableau 3. Caractéristiques sociodémographiques des enquêtées

\begin{tabular}{llc}
\hline Variables & Catégorie & Pourcentage \\
\hline Age (années) & $18-25$ & 14 \\
& $26-35$ & 24 \\
& $36-45$ & 49 \\
& $46-55$ & 10 \\
& $>56$ & 3 \\
\hline Etat civil & Mariées & 33 \\
& Célibataires & 62 \\
& Veuves & 1 \\
& Divorcées & 3 \\
\hline Niveau d'étude & Graduat & 22 \\
& Licence & 76 \\
& Master & 1 \\
& Doctorat & 1 \\
\hline Ancienneté du diplôme (depuis la fin des & $1-5$ & 19 \\
études) & $5-10$ & 48 \\
& $10-15$ & 33 \\
\hline Filières suivies à l'université & Techniques et spécialisées & 8 \\
& Sociales & 92 \\
\hline Taux d'insertion professionnelle selon la & Techniques et spécialisées \\
filière & Sociales & 60 \\
\hline
\end{tabular}

\subsection{Analyse par correspondance multiple des variables étudiées}

La figure 3 présente une analyse par correspondance multiple des variables examinées. Cette analyse met en évidence des facteurs résumant le maximum d'information en regroupant tous les éléments dans des classes homogènes c'est-à-dire les éléments qui se ressemblent ou qui s'associent. L'avantage de cette analyse est qu'elle combine les variables quantitatives et les variables qualitatives. 


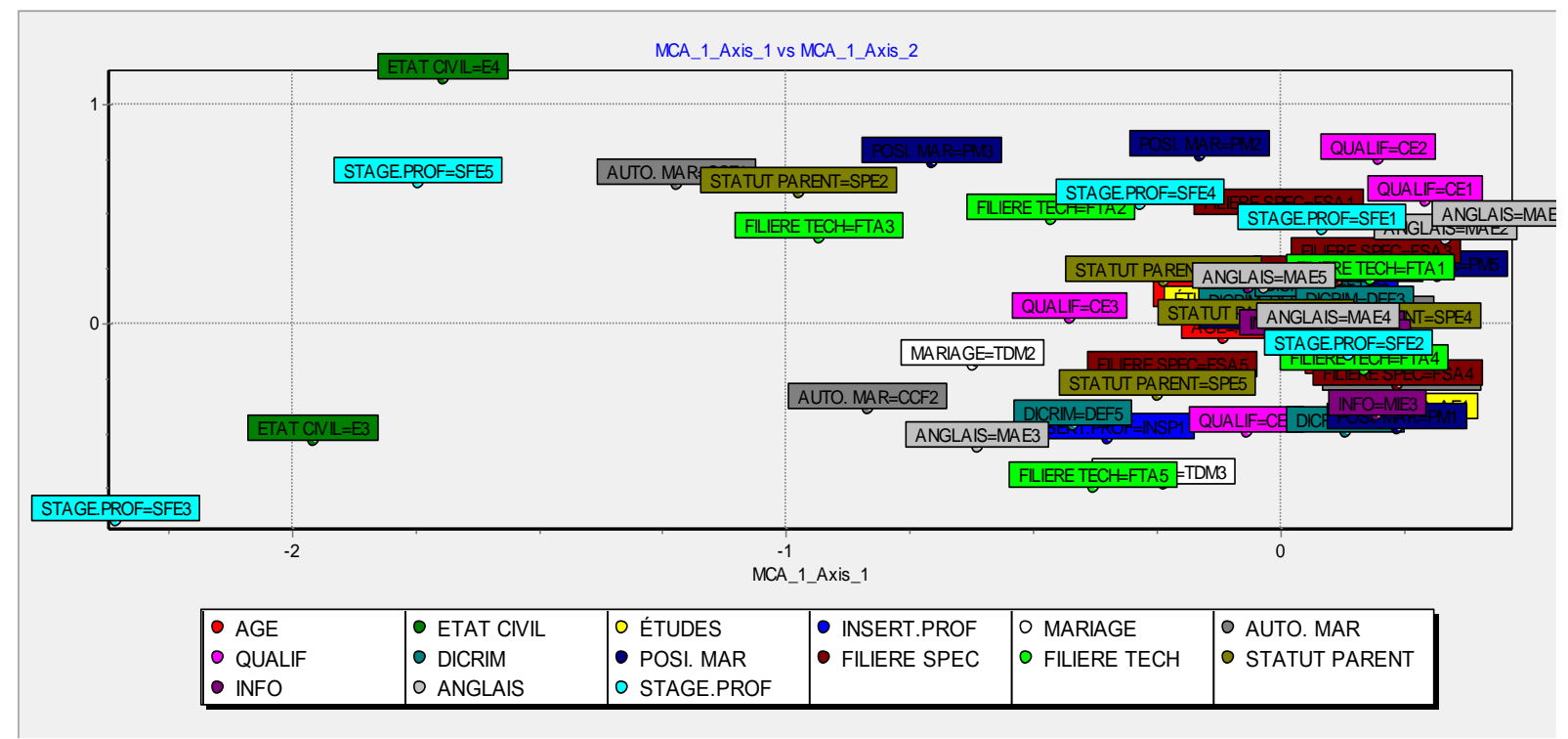

Figure 3. Analyse par correspondance multiple des variables examinées

Le tableau 4 classe les variables examinées selon leurs axes ainsi que leur niveau d'influence sur l'insertion professionnelle des femmes diplômées universitaires rencontrées. L'analyse par axe fait ressortir 3 axes des variables :

- L'axe 1 est composé de 2 variables qui touchent le statut des femmes enquêtées

- L'axe 2 est composé de 5 variables qui touchent les études des femmes enquêtées

- L'axe 3 est composé de 2 variables qui touchent l'environnement extérieur mais influencent beaucoup l'insertion professionnelle.

Tableau 4. Classement des variables par Axe selon l'ordre d'importance

\begin{tabular}{l|l|l|l|l|c}
\hline Axe 1 & $\begin{array}{l}\text { Classement } \\
\text { des } \\
\text { variables }\end{array}$ & Axe 2 & $\begin{array}{l}\text { Classement } \\
\text { des } \\
\text { variables }\end{array}$ & Axe 3 & $\begin{array}{l}\text { Classement } \\
\text { des variables }\end{array}$ \\
\hline $\begin{array}{l}\text { Autorisation } \\
\text { maritale }\end{array}$ & 18.9 & $\begin{array}{l}\text { Filière } \\
\text { Spécialisé ou } \\
\text { technique }\end{array}$ & 13.3 & $\begin{array}{l}\text { Stage } \\
\text { professionnel }\end{array}$ & 22.8 \\
\hline Mariage & 17.2 & Qualification & 11.1 & $\begin{array}{l}\text { Statut des } \\
\text { parents }\end{array}$ & 16.7 \\
\hline Position du mari & 6.3 & Informatique & 5.6 & & \\
\hline & & Anglais & 5.1 & & \\
\hline
\end{tabular}

Sur l'axe 1, on note la présence de valeurs-tests extrêmement élevées en valeur absolue, indiquant la présence de modalités significativement différentes du centre de gravité. Cet axe est composé de 2 variables qui touchent le statut des femmes enquêtées dont l'autorisation maritale, le mariage et la position du mari dans la société. L'axe 2 a permis d'observer les 5 variables qui touchent les études des femmes enquêtées. Ainsi constatons-nous que cet axe présente la filière technique, la filière spécialisée, la qualification de la femme, la connaissance de l'informatique et de la langue anglaise. L'axe 3 est composé des variables environnementales dont le statut des parents et le stage professionnel. Les résultats de la régression Stepwise (pas à pas) affichent l'existence de deux modèles qui sont statistiquement significatifs et qui expliquent respectivement à $57 \%$ et $76 \%$ la variance de l'insertion professionnelle (voir l'annexe, tableau 1). Le modèle 2 a été retenu parce qu'il exprime le plus grand score de la variance de la variable dépendante et confirme ce que l'insertion professionnelle des femmes diplômées universitaires dans le milieu professionnel à Lubumbashi. II ressort du modèle 2 que la filière spécialisée faite par la femme, la filière technique optée dans ses études, la connaissance de la langue anglaise, la position du mari ( $s$ 'il a un emploi bien rémunéré, autoriserait pas la femme de travailler), le statut des parents ( $s$ 'ils ont un poste de 
responsabilité, ils trouvent pour leur fille facilement un travail) et les compétences particulières de la femme elle-même. Le tableau 5 présente une synthèse des résultats sur les déterminants de l'insertion professionnelle au seuil de $95 \%$.

Tableau 5. Synthèse des résultats sur les déterminants de l'insertion professionnelle au seuil de $95 \%$

\begin{tabular}{|l|l|l|}
\hline \multicolumn{2}{|l|}{ Résult ats du modèle $\mathbf{2}$ avec $\mathbf{1 1}$ variables, $\mathbf{R}^{2}$ ajusté 76\%(voir annexe) } \\
\hline Facteurs déterminants & Facteurs non explicatifs & Raisons \\
\hline Filière spécialisée & L'autorisation implicite du mari & $\begin{array}{l}\text { L'homme à trop de pouvoir sur le } \\
\text { ménage }\end{array}$ \\
\hline Filière technique & La discrimination à l'embauche & $\begin{array}{l}\text { Minimisation des compétences } \\
\text { féminines, congés fréquents }\end{array}$ \\
\hline Anglais & Le mariage & $\begin{array}{l}\text { Travaux ménagers et occupation } \\
\text { des enfants }\end{array}$ \\
\hline Stage professionnel & & \\
\hline Position du mari & & \\
\hline Statut des parents & & \\
\hline Compétences & & \\
\hline
\end{tabular}

Les résultats du tableau 5 indiquent que $76 \%$ de la variabilité de la variable dépendante insertion professionnelle sont expliqués par les 10 variables explicatives. En effet, les femmes qui choisissent les études dans des filières spécialisées (médecine, infirmière, accouchement...) et des filières techniques (électricité, géologie, agronomie, électronique, chimie) sont facilement insérées dans la vie professionnelle après leurs études supérieures ou universitaires. (2)En plus, la connaissance de la langue anglaise par les femmes diplômées d'universités facilite l'insertion professionnelle (augmente les chances de la femme à trouver facilement de l'emploi) (3) La position du mari explique pourquoi certaines femmes ne cherchent pas de l'emploi. En effet, lorsque le mari a un emploi bien rémunéré, la femme ne trouve pas d'intérêt à chercher de l'emploi.(4) quant au statut des parents, cela facilite l'insertion des femmes dans la vie professionnelle en ce sens où lorsque les parents sont bien positionnés dans la société ou soit ils exercent un poste de décision dans une entreprise, ils trouvent facilement pour leurs filles de l' emploi (5) Les compétences particulières de la femme lui confère un estime de soi pour chercher du travail. Cela est aussi confirmé par Doumbia et Kuépié (2008) qui montrent que l'effet de l'éducation sur la décision d'être active s'explique par le fait qu'en accumulant du capital humain, les femmes accroissent les gains qu'elles peuvent espérer au-delà du salaire de réserve .Cette théorie est largement confirmée par les faits : les chances relatives pour une femme d'être active doublent quand on passe de sans instruction à secondaire, et sont multipliées par quatre quand elle a fréquenté l'enseignement supérieur. Le tableau 6 présente une matrice de corrélation entre les variables examinées pour en identifier toutes les variables associées positivement à l'insertion professionnelle de la femme universitaire à Lubumbashi. il ressort de ce tableau de corrélation que l'autorisation maritale, la position du mari dans la société, la connaissance de l'informatique ainsi que de l'anglais constituent des facteurs supplémentaires intéressants et importants pour l'insertion professionnelle des femmes interrogées. En RD Congo, le Code de la Famille, à ses articles 444 et 445 , confère à l'homme (époux) beaucoup de pouvoir sur son ménage, ce qui amène nombreuses femmes d'obtenir l'approbation de leurs époux avant de chercher ou contracter l'emploi ${ }^{5}$. Cependant, si l'homme est mieux rémunéré à son emploi, il a tendance à imposer à son épouse de rester à la maison, s'occuper des travaux ménagers et des enfants, malgré son niveau d'étude universitaire.

\footnotetext{
${ }^{5}$ Livre trois du code de la famille de la RD. Congo, articles 444 et 445.
} 
Tableau 6. Matrice de corrélation entre les variables examinées

\begin{tabular}{|c|c|c|c|c|c|c|c|c|c|c|c|c|c|c|c|}
\hline Variables & Age & $\begin{array}{l}\text { Etat } \\
\text { civil }\end{array}$ & Études & $\begin{array}{l}\text { Insertion. } \\
\text { Prof }\end{array}$ & Mariage & $\begin{array}{l}\text { Auto. } \\
\text { Mar }\end{array}$ & Compétence & Dicrim & $\begin{array}{l}\text { Posi. } \\
\text { Mar }\end{array}$ & $\begin{array}{l}\text { Filiere } \\
\text { spécialisée }\end{array}$ & $\begin{array}{l}\text { Filiere } \\
\text { technique }\end{array}$ & $\begin{array}{l}\text { Statut } \\
\text { parent }\end{array}$ & Info & Anglais & $\begin{array}{l}\text { Stage } \\
\text { professionnel }\end{array}$ \\
\hline AGE & 1 & $-0,091$ & 0,043 & $-0,004$ & $-0,105$ & 0,090 & $-0,030$ & $-0,010$ & 0,089 & 0,008 & 0,078 & 0,221 & $-0,057$ & 0,054 & $-0,015$ \\
\hline ETAT CIVIL & $-0,091$ & 1 & $-0,030$ & $-0,108$ & 0,092 & $-0,235$ & 0,011 & 0,126 & $-0,017$ & 0,086 & 0,090 & $-0,097$ & 0,025 & 0,031 & 0,228 \\
\hline ÉTUDES & 0,043 & $-0,030$ & 1 & $-0,022$ & $-0,041$ & 0,031 & $-0,011$ & $-0,098$ & 0,117 & $-0,081$ & $-0,103$ & $-0,029$ & 0,058 & 0,000 & $-0,076$ \\
\hline INSERT.PROF & $-0,004$ & $-0,108$ & $-0,022$ & 1 & $-0,055$ & 0,218 & $-0,254$ & $-0,081$ & 0,036 & 0,308 & 0,289 & 0,063 & 0,065 & 0,145 & $-0,015$ \\
\hline MARIAGE & $-0,105$ & 0,092 & $-0,041$ & $-0,055$ & 1 & $-0,306$ & 0,077 & 0,077 & 0,078 & 0,088 & 0,130 & $-0,149$ & 0,190 & $-0,171$ & $-0,025$ \\
\hline AUTO. MAR & 0,090 & $-0,235$ & 0,031 & 0,218 & $-0,306$ & 1 & 0,048 & $-0,112$ & $-0,005$ & $-0,111$ & $-0,071$ & 0,180 & $-0,083$ & 0,053 & $-0,514$ \\
\hline COMPTENCE & $-0,030$ & 0,011 & $-0,011$ & $-0,254$ & 0,077 & 0,048 & 1 & 0,039 & $-0,105$ & 0,207 & 0,329 & 0,004 & $-0,042$ & $-0,126$ & $-0,251$ \\
\hline DICRIM & $-0,010$ & 0,126 & $-0,098$ & $-0,081$ & 0,077 & $-0,112$ & 0,039 & 1 & $-0,033$ & 0,187 & 0,137 & $-0,010$ & $-0,056$ & $-0,063$ & $-0,031$ \\
\hline POSI. MAR & 0,089 & $-0,017$ & 0,117 & 0,036 & 0,078 & $-0,005$ & $-0,105$ & $-0,033$ & 1 & $-0,019$ & $-0,118$ & $-0,030$ & 0,119 & $-0,008$ & $-0,108$ \\
\hline FILIERE SPEC & 0,008 & 0,086 & $-0,081$ & 0,308 & 0,088 & $-0,111$ & 0,207 & 0,187 & $-0,019$ & 1 & 0,408 & 0,098 & $-0,333$ & $-0,045$ & $-0,122$ \\
\hline FILIERE TECH & 0,078 & 0,090 & $-0,103$ & 0,289 & 0,130 & $-0,071$ & 0,329 & 0,137 & $-0,118$ & 0,408 & 1 & 0,096 & $-0,138$ & $-0,193$ & $-0,103$ \\
\hline STATUT PARENT & 0,221 & $-0,097$ & $-0,029$ & 0,063 & $-0,149$ & 0,180 & 0,004 & $-0,010$ & $-0,030$ & 0,098 & 0,096 & 1 & $-0,188$ & $-0,004$ & $-0,149$ \\
\hline INFO & $-0,057$ & 0,025 & 0,058 & 0,065 & 0,190 & $-0,083$ & $-0,042$ & $-0,056$ & 0,119 & $-0,333$ & $-0,138$ & $-0,188$ & 1 & 0,030 & 0,113 \\
\hline ANGLAIS & 0,054 & 0,031 & 0,000 & 0,145 & $-0,171$ & 0,053 & $-0,126$ & $-0,063$ & $-0,008$ & $-0,045$ & $-0,193$ & $-0,004$ & 0,030 & 1 & 0,208 \\
\hline STAGE.PROF & $-0,015$ & 0,228 & $-0,076$ & $-0,015$ & $-0,025$ & $-0,514$ & $-0,251$ & $-0,031$ & $-0,108$ & $-0,122$ & $-0,103$ & $-0,149$ & 0,113 & 0,208 & 1 \\
\hline
\end{tabular}

AUTO. MAR : autorisation maritale ; DICRIM : discrimination l'embauche ; POSI. MAR :position du mari ; INFO : informatique

\section{Conclusion}

Face à la conjoncture actuelle du marché du travail, l'insertion professionnelle des femmes est devenue une problématique réelle, tant sur le plan national que sur le plan local. Les cinq dernières années, les institutions supérieures et universitaires ont diplômé plus de $36,6 \%$ des femmes à Lubumbashi mais très peu d'elles (moins de $10 \%$ ) ont été insérées dans la vie professionnelle. Dans le souci de comprendre les causes liées à ce faible taux d'insertion, une enquête couplée à une analyse des théories existantes sur l'insertion professionnelles des femmes a été privilégiée. Les résultats principaux révèlent que l'insertion professionnelle des femmes diplômées d'université est déterminée par plusieurs facteurs. II s'agit des filières suivies à l'université (filières techniques augmentent plus de chance à trouver de l'emploi que des filières sociales), la position sociale du mari ainsi que celle des parents constituent des facteurs influençant l'insertion professionnelle selon les femmes interrogées. Ce qui sous-entend que les compétences et les études faites seules ne suffisent plus à décrocher l'emploi dans le milieu de l'étude mais aussi des influences familiales, amicales ne sont pas à sous-estimer. Malgré ces influences, la connaissance de la langue anglaise et de l'informatique constitue des éléments supplémentaires positivement corrélées à l'insertion professionnelle rapide des femmes diplômées à Lubumbashi. Ces résultats nous motivent donc d'inviter et d'encourager les femmes à s'inscrire dans les filières techniques ou spécialisées tout en comprenant que l'anglais ainsi que 
l'informatique constituent actuellement des outils incontournables et nécessaires car ils peuvent aider à saisir des opportunités d'emplois dans un environnement très compétitif.

\section{Références}

[1] [1]Armstrong et Crombie (2000), Compromises in adolescents 'occupational aspirations and expectations from grade 8 to 10 ;Journal of vocational behavior, 56(1), 82-98.

[2] [2]Balasha M., Hwali M., Kesonga N., et al. (2020), Understanding the Roles of Street Vendors of Agricultural Commodities during the COVID-19 Outbreak in the Informal Economy, , Open Journal of Social Sciences 8, 115-129. https://doi.org/10.4236/jss.2020.89008.

[3] [3]Balasha, M., \& Nkulu, J. (2020). Déterminants d'adoption des techniques de production et protection intégrées pour un maraichage durable a Lubumbashi, République démocratique du Congo. Cahiers Agricultures, 29, 13. https://doi.org/10.1051/cagri/2020012.

[4] [4]Betasemi N.(2018), Vie et survie à Kinshasa: les femmes au secours des ménages, edition Lokole $n^{\circ} 206, p 56$.

[5] [5]Calmand J. et Epiphane D (2012), L'insertion professionnelle après des études supérieures :des diplômés plus égaux que d'autres... ", Formation emploi [En ligne], 117 | janvier-mars 2012, mis en ligne le 06 juin 2012, consulté le 19 avril 2019. http://journals.openedition.org/formationemploi/3509.

[6] [6]Doumbia G.,Kuépié M,(2008). Niveau et déterminants de l'insertion des femmes sur le marché du travail au Mali, STATECO N¹03.

[7] [7]lyenda, G. (2005). Street Enterprises, Urban Livelihoods and Poverty in Kinshasa. Environment\&Urbanization, 17, 56-66. https://doi.org/10.1177/095624780501700205.

[8] [8]JICA (2017). Country Gender Profile, Democratic Republic of the Congo, Final Report 53 p.

[9] [9]Kyamakosa, M. et Musewa, L. M., (2011), Le model de croissance Katangaise face a la crise fianciere mondiale; enjeux en terme d'emplois, Geneve: BIT.

[10] [10]Mellor, N.,(2014), Les jeunes diplômés universitaires: face au dilème de l'emploi.

[11] [11]Monusco, (2019), Echo de femme pour un monde 50-50, Kinshasa: Mission des Nàtion unies en RD congo.

[12] [12]Moummi, Ahmed (2010), Analyse de la pauvreté en République démocratique du Congo, Working Papers Series $N^{\circ} 112$, African Development Bank, Tunis, Tunisia.

[13] [13]Murhula B., Kitsali K., Mushagalusa B.(2020), Pauvreté et Secteur Informel à Bukavu: Profil des Détaillants des Produits Agricoles à I'Ere de la pandémie COVID-19, Journal of Economics, Finance and Accounting Studies ;2(2) :1-10.

[14] [14]Nkuku, M. Rémon(2006),Stratégies de survie à Lubumbashi (R-D Congo). Enquête sur 14000 ménages urbains, Archive congolaise, le Harmattan, Paris.

[15] [15]Nzuzi, B., 2014. microcredit et condition socioeconomique des femmes chefs de famille monoparentale dans la ville de lubumbashi quartier kisanga, Lubumbashi,p48.

[16] [16]Tambwe N. (2010). Urban agriculture and food security in the city of Lubumbashi (DRC). Doctoral dissertation, University of the Witwatersrand, 306 p. http://hdl.handle.net/10539/8176.

[17] [17]Tshomba K, Nkulu M et Kalambaie Moïse (2020) Analyse de la dépendance alimentaire aux importations des ménages dans trois communes de la ville de Lubumbashi, RDC. Int. J. of Multidisciplinary and Current research, 8(2), 389 -395.

[18] [18]UN Department of Economic and Social Affairs, Population Division.2016. The World's Cities in 2016-Data Booklet (ST/ESA/ SER.A/392).

[19] [19]Useni S, Marie A, Mahy G, Cabala K, Malaisse F, Munyemba F, et al.(2018), Interprétation paysagère du processus d'urbanisation à Lubumbashi : dynamique de la structure spatiale et suivi des indicateurs écologiques entre 2002 et 2008. In Bogaert J, Colinet G, Mahy G, eds. Anthropisation des paysages katangais. Gembloux, Belgique : Presses Universitaires de Liège - Agronomie, pp. $281-296$.

[20] [20] PNUD (2014). Pérenniser le progrès humain : réduire les vulnérabilités et renforcer la résilience, Rapport sur le Développement Humain, Programme de Nations Unies pour le Développement, 1 UN Plaza, New York, NY 10017, USA, 259p. 


\section{Annexes}

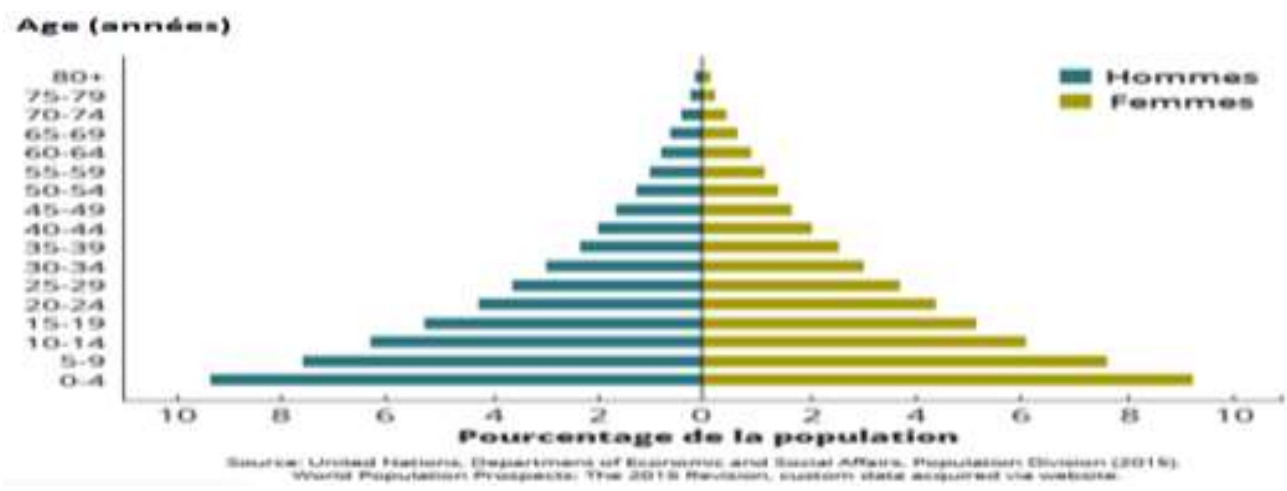

Tableau 1. Régression de la variable Insertion Professionnelle

\begin{tabular}{|c|c|c|c|}
\hline \multicolumn{2}{|c|}{ Modèle 1 de régression } & \multicolumn{2}{|c|}{ Modèle 2 de régression } \\
\hline \multicolumn{2}{|c|}{ Coefficients d'ajustement (INSERT.PROF) } & \multicolumn{2}{|c|}{ Coefficients d'ajustement (INSERT.PROF) } \\
\hline Observations & 384 & Observations & 384 \\
\hline Somme des poids & 384 & Somme des poids & 384 \\
\hline $\mathrm{R}^{2}$ & 0,582 & $\mathrm{R}^{2}$ & 0,786 \\
\hline $\mathrm{R}^{2}$ ajusté & 0,571 & $\mathrm{R}^{2}$ ajusté & 0,765 \\
\hline MCE & 0,111 & MCE & 0,091 \\
\hline RMCE & 0,333 & RMCE & 0,301 \\
\hline $\mathrm{Cp}$ & 25 & $\mathrm{Cp}$ & 41 \\
\hline $\mathrm{AIC}$ & $-819,746$ & AIC & $-883,438$ \\
\hline SBC & $-720,980$ & SBC & $-721,461$ \\
\hline PC & 0,897 & PC & 0,761 \\
\hline
\end{tabular}

\section{Équation du modèle 2} (INSERT.PROF) :

INSERT.PROF $=1,006-6,593 *$ MARIAGE-TDM1+0,386*AUTO. MAR-CCF 1-0,214*AUTO CCF2-0,343*AUTO. MAR-CCF4+0,188*QUALIF-CE $1+9,87 *$ QUALIF-CE $2+0,181 * Q U A$ CE3+0,141*QUALIF-CE4+9,252*DICRIM-DEF $1+0,127 *$ DICRIM-DEF2-0,122*DICRI DEF3+7,840*DICRIM-DEF4-8,772*POSI. MAR-PM1-8,463*POSI. MAR-PM2-0,687*POSI PM3-0,101*POSI. MAR-PM4+0,205*FILIERE SPEC-FSA 1+0,145*FILIERE SPECFSA $2+0,116 *$ FILIERE SPEC-FSA $3+6,318 *$ FILIERE SPEC-FSA 4+0,201*FILIERE TEC FTA $1+0,207^{*}$ FILIERE TECH-FTA $2-2,459^{*}$ FILIERE TECH-FTA $3+0,166^{*}$ FILIERE TEC FTA4+2,593*STATUT PARENT-SPE1+0,184*STATUT PARENT-SPE2+0,083*STATU PARENT-SPE3+7,624*STATUT PARENT-SPE4+0,103*INFO-MIE 1+0,145*INFO-MIE 3,328*INFO-MIE3+0,0463*INFO-MIE4-7,895*ANGLAIS-MAE1-7,599*ANGLAIS-MA $0,176 *$ ANGLAIS-MAE3-1,692*ANGLAIS-MAE4+0,572*STAGE.PROF-

SFE $1+0,602 *$ STAGE.PROF-SFE $2+0,606 *$ STAGE.PROF-SFE $3+0,591 *$ STAGE.PROF-SF 